\title{
VALIDEZ DE UN INSTRUMENTO MULTIDIMESIONAL PARA MEDIR FACTORES DE RIESGO ASOCIADOS A TRASTORNOS DE LA CONDUCTA ALIMENTARIA EN PÚBERES MEXICANOS
}

\section{VALIDITY OF A MULTIDIMENSIONAL QUESTIONNAIRE TO MEASURE RISK FACTORS ASSOCIATED TO EATING DISORDERS IN MEXICAN PUBESCENTS}

\author{
Teresita de Jesús Saucedo-Molina (1), Claudia Unikel-Santoncini (2) \\ (1) Área Académica de Nutrición, Instituto de Ciencias de la Salud, \\ Universidad Autónoma del Estado de Hidalgo. Hidalgo, México. \\ (2) Dirección de Investigaciones Epidemiológicas y Psicosociales, \\ Instituto Nacional de Psiquiatría Ramón de la Fuente Muñiz, Distrito Federal, México.
}

\begin{abstract}
Objective: To obtain the validity and internal consistency of a questionnaire for the screening of risk factors associated to eating disorders in Mexican pubescent males and females. Subjects and methods: The questionnaire was applied to a non-probabilistic sample of 504 males and 511 female students in Mexico City from 11 to 15 years of age with a median of 12,13. Statistical methods included internal consistency analysis (Cronbachís alpha) and principal components factor analysis with oblimin rotation. Results: Both instruments obtained fivefactors structure, showed a good total Cronbachís alpha score: males 0,75 (55,6 \%), and females 0,83 (57,6\%) of the total explained variance. Males displayed a normal eating behavior factor, while for females a restrictive dieting factor was obtained. Conclusions: This questionnaire is a valid measure in pubescent boys and girls for assessing risk factors associated with eating disorders, in particular sociocultural factors, eating behaviors and pubertal development.
\end{abstract}

Key words: pubescent; eating disorders; validity; Mexico.

Este trabajo fue recibido el 2 de Marzo de 2009 y aceptado para ser publicado el 15 de Diciembre de 2009.

\section{INTRODUCCIÓN}

Es conocido que la etiología de los trastornos de la conducta alimentaria es multicausal (1) entre ellos: factores genéticos (2-4), familiares (5-8), socioculturales (9-16), psicológicos (17-20), y ciertos patrones alimentarios (21-23), muchos de ellos considerados como de riesgo, y altamente relacionados unos con otros.

Son factores de riesgo aquellos que aumentan la probabilidad de que se altere la salud y se desencadene alguna enfermedad. También se les denomina así, porque tienen un efecto negativo en la salud del individuo provocando un desequilibrio ya que hacen al organismo vulnerable facilitando la aparición de la enfermedad (24). Los grupos más vulnerables a estos factores de riesgo asociados con los trastornos de la conducta alimentaria sobresalen son los púberes (25-27), especialmente porque se encuentran en una etapa de cambios corporales y además están en la búsqueda de identidad y de cumplir con las normas establecidas por aquellos a quienes admiran y esperan de ellos determinadas conductas $(28,29)$.

Estudios realizados en púberes de ambos sexos han revelado que muchos de ellos están preocupados con respecto a su imagen corporal, los alimentos y el control de peso (30). De igual manera se ha encontrado que niñas de 9 a 12 años hacen dieta restringida para reducir peso y mejorar la figura corporal (31).

Resulta importante resaltar que se han identificado diferencias importantes en la manera en que las púberes 
y los púberes, expresan y viven la preocupación por su figura, el peso y la alimentación. En un estudio realizado en 457 niños y niñas entre 9 y 11 años de edad que acudían a 10 escuelas rurales en el centro de Iowa, se reveló que muchos de ellos estaban preocupados por estos aspectos pero que las niñas expresaron mucho más el deseo de ser delgadas en comparación con los niños (60.3\% vs. $38.4 \%)$, mientras que los niños querían ser más altos que las niñas (67,2\% vs. 49,1\%) (30).

En diversas investigaciones mexicanas se ha demostrado, de manera concordante con las efectuadas en otros países, que la preocupación por el peso y la imagen corporal, así como modificaciones drásticas en el patrón alimentario, están presentes en la pubertad. En una de estas investigaciones, en una muestra de 962 mujeres de 9 a 13 años, se detectaron factores de riesgo asociados con el desarrollo de trastornos de la alimentación. Los hallazgos mostraron que casi la mitad de las púberes estaban insatisfechas con su imagen corporal. Asimismo, se confirmó que en edades de 11 a 13 años, ya está presente, en una proporción mayoritaria (94\%) la aceptación del estereotipo social de la cultura de la delgadez, centrado en la idea de que un cuerpo delgado es el determinante del atractivo de la mujer. También se encontró que las tres cuartas partes de la muestra respondieron estar preocupadas (mucho y demasiado) por su peso corporal, no obstante que muchas de ellas eran de peso normal o incluso de peso bajo (14).

Un estudio realizado por Gómez Pérez-Mitré y cols (26) en una muestra de 200 púberes mexicanos con edad promedio de 10.8 años, mostraron que $51 \%$ de las mujeres y $54 \%$ de los hombres, manifestaron insatisfacción por su figura corporal, donde las mujeres querían ser más delgadas y los varones más robustos (musculosos).

Otro estudio de tipo exploratorio, realizado en 200 escolares mexicanos, hombres y mujeres con edades de 9 a 13 años, concluyó que los hombres, realizan más número de comidas al día al compararlos con las mujeres, hallazgo que confirmó dos cosas, que la demanda energética de los varones es mayor y su preocupación por el peso corporal es menor en el sentido de adelgazar (32).

En 437 escolares mexicanos con edad promedio de 10.8 años, se demostró que 25\% aceptó estaba haciendo dieta con propósitos de control de peso. En esta misma muestra se registró que más mujeres que hombres omitían un alimento del día (en general, la cena) y consumían menor proporción de alimentos considerados por ellos como que engordan tales como tortillas, pan, y frijoles, con la intención de bajar o controlar su peso corporal (33).

Resulta cada vez más evidente que los púberes están adquiriendo la preocupación de los adultos con relación a los alimentos, la obsesión por un peso bajo y la tendencia a querer alcanzar una figura delgada idealizada. Esta ansiedad se traduce cada vez más en conductas de riesgo que pueden favorecer el surgimiento de un trastornos alimentario, los cuales tiene importantes consecuencias físicas, biológicas y psicológicas, y peligrosa, si se siguen dichas conductas en etapas cruciales de la vida como lo es la pubertad.

De aquí la necesidad de contar con instrumentos válidos a través de los cuales sea posible tamizar púberes en riesgo de desarrollar un trastorno alimentario, que permitan diseñar intervenciones preventivas y eficaces de promoción de la salud

En la literatura, se han diseñado diversos instrumentos para identificar niños y adolescentes en riesgo de desarrollar un trastorno de esta naturaleza. Uno de ellos, es el cuestionario alemán sobre conductas alimentarias, conocido como el DEBQ (Dutch Eating Behaviour Questionnaire, y cuya estructura consiste de tres subescalas que miden: dieta restringida, alimentación emocional y control alimentario externo (34). El DEBQ fue diseñado originalmente para adultos, pero ha sido utilizado y validado en muestras de niños de 7 a 9 años de edad (35-37)

Otro instrumento localizado en la literatura es el CEBI (Children's Eating Behavior Inventory), diseñado para niños de 2 a 10 años de edad, y cuya finalidad es la de evaluar la conducta alimentaria y los problemas durante las comidas en este tipo de población. Fue desarrollado sobre la teoría transaccional de sistemas que trata de explicar la relación padres-hijos dentro del contexto de la alimentación. Resulta evidente el que este instrumento es para niños y no precisamente enfocado hacia conductas alimentarias asociadas a trastornos alimentarios, sino más bien a problemas alimentarios típicos de preescolares y escolares y lo que estos problemas significan para los padres (38).

El denominado Children's Eating Attitudes Test (ChEAT) (39), versión para niños del Eating Attitud Test (EAT) (40), es empleado para medir una variedad de actitudes y conductas asociadas con anorexia y bulimia nervosa. Incluye una escala de 26 reactivos que miden dieta restringida, preocupación por la comida y purga. A pesar de que ha sido sometido a algunos análisis factoriales (41), se requieren más investigaciones sobre su validez.

Por último se encuentra el KEDS (Kid's Eating Disorders Survey) diseñado para medir síntomas de trastornos alimentarios en niños, consta de 12 preguntas acerca de la insatisfacción con el peso, percepción de la imagen corporal, dieta restringida y conductas purgativas 
(42). Este cuestionario está en sus etapas tempranas de desarrollo psicométrico.

Aunque algunos de estos instrumentos han sido validados, resulta evidente que no es recomendable emplearlos en púberes, ya que ellos tienen otro nivel de comprensión, y de habilidades cognoscitivas, además de que en esta etapa comienza a haber una mayor preocupación por la apariencia y el peso corporal. Debe señalarse, que ninguno de ellos considera aspectos socioculturales como factores de riesgo asociados a los trastornos alimentarios, ni tampoco la conducta alimentaria normal, la cual debería ser un factor protector dentro del contexto de estas patologías y ninguno de ellos ha sido validado en México en este grupo etario.

Para cubrir estas carencias surgió el objetivo del presente estudio, el cual consistió en mostrar la consistencia interna la validez de constructo de un breve instrumento, adaptado específicamente para púberes mexicanos, de tipo autoaplicable, tanto para hombres (FRATAP-H), como para mujeres (FRATAP-M), que midiera conductas alimentarias y aspectos socioculturales, con la finalidad de ser utilizado como prueba de tamizaje en este tipo de población. Este instrumento proviene de una modificación de dos cuestionarios, el EFRATA (Escala de Factores de Riesgo Asociados a Trastornos Alimentarios) $(43,44)$ y el CIMEC (Cuestionario de Influencias sobre el Modelo Estético Corporal) (45) ambos validados en mujeres hispanas, específicamente mexicanas y españolas, respectivamente, empleados para medir factores de riesgo asociados a trastornos de la conducta alimentaria.

\section{SUJETOS Y MÉTODO Sujetos}

Se llevó a cabo un estudio de campo de tipo transversal, en una muestra total intencional, con selección no probabilística constituida por 1306 sujetos, estudiantes de quinto y sexto grado de primaria y de primero y segundo grado de secundaria, de 8 escuelas mixtas, tanto públicas (58\%) como privadas (42\%) del Sur de la Ciudad de México, con edades entre 11 a 15 años, una media de 12,13 ( $\mathrm{DE}= \pm 1,23)$. Se obtuvieron dos submuestras: 1) hombres $(\mathrm{N}=674)$ estudiantes de escuelas públicas (54\%) y privadas (46\%), con una media de $12,54(\mathrm{DE}$ $= \pm 0,96): 2)$ mujeres $(\mathrm{N}=632)$ estudiantes de escuelas públicas $(60 \%)$ y privadas $(40 \%)$, con una media de $12,08(\mathrm{DE}= \pm 1,3)$.

\section{Instrumento}

Partiendo de que existen diferencias conceptuales por sexo sobre el peso, la figura, y la alimentación, para la estructuración inicial del instrumento se realizaron grupo focales. Para formarlos se reclutaron 16 escolares, 8 hombres y 8 mujeres, estudiantes de quinto y sexto grado de primaria, con edades de 10 a 12 años. Los sujetos fueron elegidos mediante un muestreo aleatorio simple a partir de la lista de asistencia, de la que se eligió al azar a cuatro hombres y cuatro mujeres de cada grado. La finalidad de estos grupos fue la de presentarles los reactivos que integraban cada instrumento para que emitieran su opinión sobre los mismos, eligieran las preguntas y determinaran si eran comprendidas por ellos o no. Con base en lo arrojado en estos grupos focales, en los que se trabajó separando a los sujetos por sexo, se eligieron 26 reactivos del EFRATA los cuales fueron presentados en la penúltima sesión focal para ser analizados y discutidos nuevamente, y decidir junto con el grupo si quedaban igual o debían ser modificados o eliminados. En la última sesión se hizo lo mismo con el CIMEC-26, instrumento que evalúa la influencia de factores socioculturales sobre el modelo corporal. Para la estimación de la madurez sexual se añadieron algunas preguntas específicas sobre el tema, además de haberse empleado imágenes representando los principales estadios de Tanner (46), mismos que se muestran en el cuadro 1.

La versión final del instrumento para púberes, en su modalidad para hombres y para mujeres, quedó formado por: 19 reactivos del EFRATA; 4 sobre madurez sexual; y 12 para medir los factores socioculturales. La calificación de las respuestas se distribuyó en cuatro categorías: 1 (nunca); 2 (pocas veces); 3 (muchas veces); 4 (siempre). Se añadieron 8 reactivos para recopilar información sobre aspectos sociodemográficos.

\section{Procedimiento}

Previa aprobación del proyecto por los directivos de cada escuela, y con su autorización, se procedió a la aplicación grupal de los cuestionarios, durante el horario regular de clases. Esta actividad fue llevada a cabo por personas previamente capacitadas, quienes dieron exactamente las mismas instrucciones de llenado a cada grupo.

Se hizo una presentación de los aplicadores, posteriormente se explicó a los alumnos el objetivo del estudio, la importancia de su participación voluntaria, la confidencialidad de sus respuestas y que cualquier duda sería aclarada en ese momento con sólo levantar su mano. Una vez comprendido y aceptado por los alumnos, los cuestionarios fueron distribuidos en el aula y respondidos por ellos. A cada sujeto se le asignó un código con el propósito de mantener su anonimato, para posteriormente poder elaborar el banco de datos.

Se debe remarcar el hecho de que la presente investigación de acuerdo a lo establecido en el Reglamento 
de la Ley General de Salud, en su apartado sobre investigación en seres humanos (47) se considera de riesgo mínimo, ya que se emplean para el levantamiento de los datos, procedimientos comunes tanto físicos (pesar y medir), como psicológicos (cuestionario) en los que no se manipula la conducta del sujeto de investigación, por lo cual no se requirió del consentimiento informado por escrito de los participantes.

Para la elaboración del banco de datos y el análisis del mismo se empleó el programa de cómputo SPSS para Windows, versión 11(48).

\section{RESULTADOS}

Se eliminaron todos aquellos cuestionarios que no fueron contestados en su totalidad, de tal manera que de las submuestras originales quedaron 504 cuestionarios de hombres y 511 de mujeres, lo cual representó una pérdida del 22,3\% de la muestra inicial.

Una vez que se tenía el banco de datos totalmente limpio, se procedió a un análisis de consistencia interna de la escala, considerando como válidos los valores de las correlaciones ítem-total iguales o mayores a 0,30.

Posteriormente cada instrumento fue sometido a un

\section{CUADRO 1}

Dibujos sobre los estadios de Tanner empleados para evaluar el desarrollo puberal.

11.-Observa con cuidado las siguientes figuras de niño. Como puedes ver cada una tiene una letra. Escoge aquella figura (sólo una) que refleje más tu desarrollo actual marcando con una cruz $(X)$ la letra correspondiente.

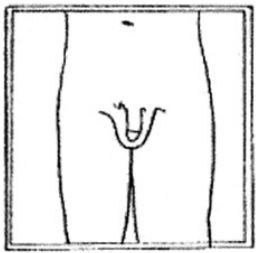

(A)

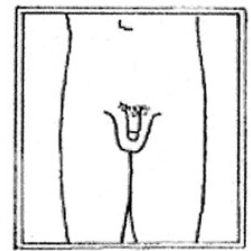

(B)

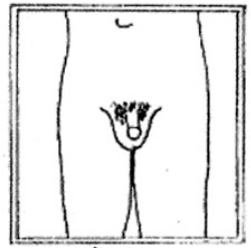

(c)

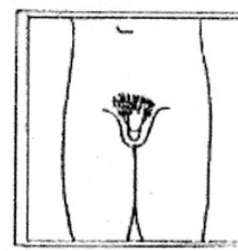

(D)

11.-Observa con cuidado las siguientes figuras de niña. Como puedes ver cada una tiene una letra. Escoge aquella figura (sólo una) que refleje más tu desarrollo actual marcando con una cruz $(\mathrm{X})$ la letra correspondiente.

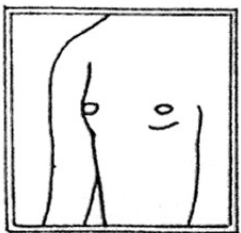

(A)

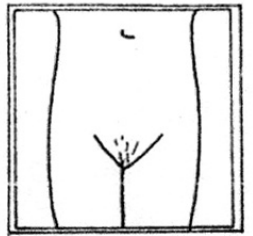

(A)

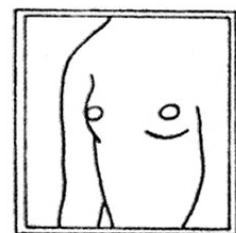

(B)

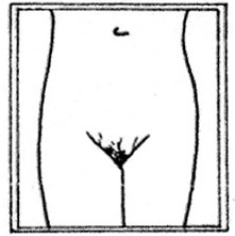

(B)

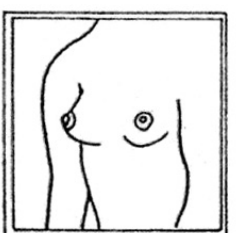

(c)

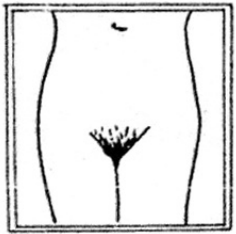

(c)

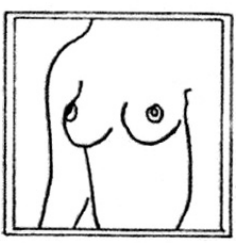

(D)

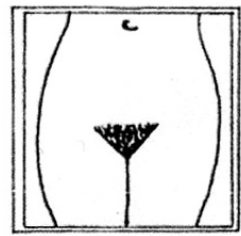

(D) 
análisis factorial de Máxima verosimilitud con rotación Oblimin. Se aceptaron como factores solamente aquellos que obtuvieron un mínimo de tres reactivos y una carga factorial igual o mayor a 0,40 (49).

\section{Instrumento de hombres}

El instrumento final quedó formado por 22 reactivos, agrupados en 5 factores, después de 5 iteraciones, que explicaron el 55,6\% de la varianza, mismo que alcanzó un valor de consistencia interna alfa de Cronbach de 0,75 .

Los cinco factores puros obtenidos del análisis en la submuestra de hombres, fueron: Factor 1, Influencia de la publicidad con 5 reactivos y un valor de alfa de $=$ 0,87; Factor 2, conducta alimentaria normal integrado por 6 reactivos mismo que alcanzó un alfa de 0,72; Factor 3, denominado malestar por la imagen corporal y formado por 5 ítems, obtuvo un alfa de 0,68; Factor 4: desarrollo puberal con 3 reactivos alcanzó un alfa $=$ 0,72 y por último el factor 5 , en el que se agruparon 3 reactivos, y al que se le denominó tiempos de comida, registró un valor de alfa de 0,66 . Debe aclararse que un factor puro, es aquél en el que los reactivos agrupados cargaron exclusivamente en dicho factor.

En la tabla 1 se muestra la distribución de los reactivos por factor junto con su carga factorial, valores propios, porcentaje de varianza explicada y el valor alfa de Cronbach alcanzada por factor.

\section{Instrumento de mujeres}

Quedó integrado por 23 reactivos, el análisis factorial permitió obtener un modelo final constituido por 5 factores puros, después de 6 iteraciones, que explicaron el 57,6\% de la varianza, arrojando un valor alfa de Cronbach de 0,83 .

Los factores finales fueron: Factor 1, denominado influencia de la publicidad, agrupó 6 reactivos mismos que alcanzaron un valor alfa de 0,87 ; Factor 2, llamado desarrollo puberal compuesto por 3 reactivos mismos que alcanzaron un alfa $=0,79$; Factor 3 , Tiempos de comida con 3 reactivos, y un valor de alfa $=0,79$; Factor 4 designado malestar con la imagen corporal, que quedó integrado por 5 reactivos y con alfa de 0,72 ; y finalmente el Factor 5, nombrado dieta restringida, mismo que alcanzó un alfa de 0,71 con 6 reactivos.

En la tabla 2 se muestra cada factor con sus valores propios, porcentajes de varianza explicada y el valor de alfa de Cronbach alcanzado.

\section{DISCUSIÓN}

Se debe resaltar que la presencia de algunos signos y síntomas de los trastornos alimentarios no son un indicador seguro del desarrollo de la enfermedad, sí de alguna manera señalan a los grupos e individuos con mayor riesgo de adquirirla. Además, desde el punto de vista epidemiológico, el detectar tempranamente cualquier sintomatología permite intervenir precozmente limitando o evitando los daños causados por la psicopatología (50)

Resulta interesante cómo con las mismas preguntas, se formaron diferentes factores en ambos grupos. Por ejemplo, en los varones no apareció el factor denominado dieta restringida, pero apareció otro, que en las mujeres no, al cual se le dio el nombre de conducta alimentaria normal. Al parecer estos resultados coinciden con algunas investigaciones realizadas en México, donde se ha confirmado que los hombres tienden a comer más, y les preocupa menos la figura y el peso corporal en el sentido de ser más delgados, a diferencia de las mujeres (32).

En las mujeres se corrobora una mayor inclinación a la preocupación por la delgadez, el peso y la figura, que al comer adecuadamente, y que para ellas la dieta restringida es un concepto claramente identificado y normativo desde edades muy tempranas, hallazgos que también concuerdan con la creencia de que comer poco es más femenino (51).

En ambos grupos, el primer factor obtenido fue el de la influencia que la publicidad tiene sobre el modelo estético corporal, lo cual coincide con diversos estudios tanto nacionales como internacionales (9-12, 15-16, 52-53), en donde se ha visto que un factor de gran peso para el seguimiento de conductas alimentarias de riesgo es la publicidad de productos orientados a la promoción de la delgadez, el moldeamiento de la figura corporal y el combate de la obesidad.

El principal aporte de este estudio, es proporcionar un instrumento válido y breve, específicamente para ser utilizado en púberes de ambos sexos que permita medir factores de riesgo asociados con el surgimiento, mantenimiento y desarrollo de los trastornos de la conducta alimentaria, específicamente factores socioculturales, conductas alimentarias y desarrollo puberal .

La insatisfacción con la imagen corporal se relaciona con el surgimiento y mantenimiento de los trastornos de la conducta alimentaria. La mayoría de los investigadores distinguen dos componentes en dicha variable, la perceptual y la actitudinal o afectiva. La perceptual consiste en un inadecuado juicio del propio tamaño corporal, y es medida a través de escalas que van desde 5 hasta 12 dibujos del cuerpo humano. El componente actitudinal o afectivo consiste en la insatisfacción con el tamaño del cuerpo, la figura, o cualquier otro aspecto referente a la apariencia física. A pesar de que muchas son las investigaciones que consideran el componente 


\section{TABLA 1}

\section{Resultado del análisis factorial del cuestionario para medir factores de riesgo asociados a trastornos alimentarios en púberes hombres}

\section{Factor}

\section{Cargas factoriales}

\section{I) Influencia de la publicidad}

Llaman tu atención los anuncios de televisión sobre productos para adelgazar.

Te llaman la atención los anuncios de productos y reportajes

para adelgazar que aparecen en las revistas.

Llaman tu atención los anuncios de productos para adelgazar

de farmacias y tiendas.

Te interesan los artículos, programas de radio y/o televisión

relacionados con el control de la obesidad.

Te llaman la atención las conversaciones o comentarios acerca del peso, dietas y la figura del cuerpo.

\section{II) Conducta alimentaria normal}

Cuidas que tu dieta sea nutritiva.

Comes lo que es bueno para tu salud

Cuidas lo que comes para no subir de peso.

0.639

Procuras mejorar tus hábitos alimentarios.

0.608

Procuras comer verdura.

0.574

Cuidas que tus comidas contengan alimentos con fibra.

\section{III) Malestar por la imagen corporal}

Llaman tu atención los anuncios de televisión o revistas que tratan sobre como volverse un hombre musculoso.

Te gustaría tener el cuerpo de Arnold Scharzenegger,

Rambo o Guardianes de la Bahía.

Envidias el cuerpo de los modelos que aparecen en los desfiles de modas o en los anuncios de ropa.

Te da envidia el cuerpo de gimnastas, nadadores, bailarines

y otros deportistas.

0.654

Te gusta el cuerpo delgado de las artistas de televisión.

0.598

\section{IV) Pubertad}

Desarrollo de vello puberal. (Imagen).

Tienes vello en tus axilas.

El sonido de tu voz ha cambiado.

\section{V) Tiempos de comida}

Acostumbras realizar tus tres comidas.

Acostumbras cenar o merendar.

Acostumbras desayunar.

Número de factor

Valores propios

Porcentaje de varianza explicada

Valor alfa de Cronbach)

$\begin{array}{cc}\text { I } & \text { II } \\ 3.45 & 2.61 \\ 15.7 & 11.9 \\ 0.87 & 0.72\end{array}$

III
2.39
10.9
0.73

IV

V

$1.98 \quad 1.81$

Rotación oblimin con el método de extracción de máxima verosimilitud (Maximum Likelihood) 
TABLA 2

\section{Resultado del análisis factorial del cuestionario para medir factores de riesgo asociados a trastornos alimentarios en púberes mujeres.}

\section{Factor}

Cargas factoriales

\section{I) Influencia de la publicidad}

Llaman tu atención los anuncios de televisión sobre productos para adelgazar.

Llaman tu atención los anuncios de productos para

adelgazar de farmacias y tiendas.

Te interesan los artículos, programas de radio y/o televisión

relacionados con el control de la obesidad.

Te llaman la atención las conversaciones o comentarios acerca del peso, dietas y la figura del cuerpo.

Llama tu atención los anuncios de televisión o revistas que tratan sobre como moldear la figura.

Te llaman la atención los anuncios de productos y reportajes para adelgazar que aparecen en las revistas.

\section{II) Malestar por la imagen corporal}

Te gustaría tener el cuerpo de Britney Spears, Anahí o Thalía.

Te gusta el cuerpo delgado de las artistas de televisión.

0.749

0.737

0.734

0.685

0.404

Te molesta que te digan que estás llenita o gordita o cosas parecidas aunque sea un comentario en broma

0.656

0.654

0.647

0.558

0.533

0.449

Estas a dieta para bajar de peso, la rompes y la vuelves a empezar.

\section{IV) Pubertad}

Desarrollo de vello puberal. (Imagen)

Tienes vello en tus axilas.

\section{V) Tiempos de comida}

Acostumbras realizar tus tres comidas.

Acostumbras desayunar.

Acostumbras cenar o merendar.

\section{Número de factor}

Valores propios

Porcentaje de varianza explicada

Valor alfa de Cronbach

$\begin{array}{cc}\text { I } & \text { II } \\ 3.69 & 2.78 \\ 16.08 & 12.08 \\ 0.87 & 0.79\end{array}$

\section{III}

IV

2.44

2.16

VI

10.6

2.16

0.87

0.79

9.4

0.72

Rotación oblimin con el método de extracción de máxima verosimilitud (Maximum Likelihood) 
perceptual, hay una mayor consistencia en los hallazgos obtenidos a partir de la medición del componente actitudinal o afectivo (54).

Otro de los aportes de este estudio, proviene de que es el primer instrumento, que mide en púberes la insatisfacción con la imagen corporal, desde una dimensión actitudinal o afectiva, a través del factor denominado malestar con la imagen corporal. Debe hacerse hincapié que en este factor en el reactivo donde se pregunta: ¿Te gustaría tener el cuerpo de, y se añade el nombre específico de algunos artistas y figuras importantes en el medio del espectáculo, se pueden poner nombres más actuales, e incluso personalidades de la región o país en donde se pretenda aplicar el cuestionario. Otro de los aportes es el haber incluido dentro del instrumento, la madurez sexual o desarrollo puberal, factor considerado por algunos investigadores como precipitante de los trastornos alimentarios $(25,51)$.

La limitación primordial de esta investigación, es la de no poder generalizar sus resultados debido a que no se trabajó con una muestra representativa de la población. Sin embargo, si permite un primer acercamiento con respecto a las tendencias en dicho grupo. Debe señalarse que este trabajo es la continuación del primer estudio realizado sobre las propiedades psicométricas de los instrumentos (55), lo que compromete a las investigadoras a profundizar no solamente en la validez y consistencia interna de los mismos, sino también sobre su confiabilidad.

\section{RESUMEN}

Objetivo: obtener la validez y consistencia interna de un cuestionario para púberes mexicanos, hombres y mujeres, que evalúe factores de riesgo asociados a trastornos de la conducta alimentaria. Sujetos y método: El cuestionario se aplicó a una muestra no probabilística, de púberes estudiantes de la ciudad de México (504 hombres y 511 mujeres), con edades de 11 a 15 años de edad, con una media de 12,13 . Se realizó un análisis de consistencia interna (alfa de Cronbach) y un análisis factorial de componentes principales con rotación oblimin por el método de máxima verosimilitud. Resultados: Ambos instrumentos arrojaron 5 factores, con una buena consistencia interna, alcanzando un valor total de alpha de Cronbach de 0,75 para hombres $(55,6 \%$ de varianza explicada) y 0,83 para mujeres $(57,6 \%$ de varianza explicada). Conclusión: El instrumento resultó válido en hombres y mujeres, para medir factores de riesgo asociados a trastornos alimentarios, principalmente socioculturales, conductas alimentarias y desarrollo puberal.

Palabras clave: púberes; trastornos alimentarios; validez; México.

Dirigir la correspondencia a:

Dra.

Claudia Unikel-Santoncini

Dirección de Investigaciones

Epidemiológicas y Psicosociales

Instituto Nacional de Psiquiatría

Ramón de la Fuente Muñiz

Calzada Xochimilco 101,

Huipulco, Tlalpan 14370

México, DF.

E-mail: unikels@imp.edu.mx

Agradecimientos: Al Consejo Nacional de Ciencia y Tecnología (CONACYT) por haber aportado los recursos económicos para realizar la presente investigación. A la Dra. Gilda Gómez-Peresmitré, profesora-investigadora de la Facultad de Psicología en la Universidad Nacional Autónoma de México, y al Dr. Joseph Toro, profesor hemérito en la División de Ciencias de la Salud de la Facultad de Medicina, en la Universidad de Barcelona, por su excelente disposición al haber permitido el uso y modificación de sus respectivos cuestionarios.

\section{BIBLIOGRAFÍA}

1. Bruce B. Wilfley D. Binge eating among the overweight population: A serious and prevalent problem. J Am Dietetic Assos. 1996; 96 (1): 58-61.

2. Kipman A. Gorwood P. Mouren-Siméoni M.C. Adès J. Genetic factors in anorexia nervosa. Europ Psychiatry 1999;14: 189-198.

3. Klump L.K. Kaye H.W. Strober M. The envolving genetic foundations of eating disorders. Psychiatr Clin North Am 2001;24 (2): 215-225.

4. Strober M. Freeman R. Lampert C. Diamond J. Kaye W. Controlled family study of anorexia nervosa and bulimia nervosa: evidence of shared liability and transmission of partial syndromes. Am J Psychiatr 2000; 157(3): 393-401.

5. Bruch H. Eating disorders: Obesity, Anorexia nervosa and the person within. Basic Books, New York, 1973.

6. Bruch H. Thin and fat people. Kaplan J.R. (Ed.) A woman's conflict: The special relationship between women and food. Englewood Cliffs, Prentice Hall, New Jersey, 1980.

7. Garfinkel P.E. Rose D.M. Darby P.L. Branders J.S. O'Hanlon J. Walsh N. A comparision of characteristics in families of patient with anorexia nervosa and normal controls. Psychol Med 1983; 13: 821-828.

8. Edmunds H. Hill A.J. Dieting and the family context 
of eating in young adolescents children. Int $\mathrm{J}$ Eat Disord 1999;25: 435-440.

9. Toro J. Cervera M. Pérez P. Body shape, publicity and anorexia nervosa. Social Psychiat Epidem 1988; 23: 132-135.

10. Toro J. Factores socioculturales en los trastornos de la ingestión. Anuario de Psicología 1989; 38 (1): 25-47.

11. Martínez M.E. Toro J. Salamero M. Blecua M.J. Zaragoza M. Influencias socioculturales sobre las actitudes y conductas femeninas relacionadas con el cuerpo y la alimentación. Rev Psiquiatría Fac Med Barna. 1993; 20(2): 51-65.

12. Martínez M.E. Toro J. Salamero M. Influencias socioculturales favorecedoras del adelgazamiento y sintomatología alimentaria. Rev Psiquiatría Fac Med Bana 1996; 23(5): 125-133.

13. Ritter M. Mothers, daughters, culture and criticism. An examination of eating problems in adolescent girls. Dissertation Abstracts International: Section B: The Science and Engineering 1998; 58 (9-B): 5138 .

14. Gómez Pérez-Mitré, G. Preadolescentes Mexicanas y la Cultura de la Delgadez: Figura Ideal Anorética y Preocupación Excesiva por el Peso Corporal. Rev Mex Psicol 1999; 16 (1): 153-165.

15. Saucedo-Molina T. Cantú Gutiérrez N. Trastornos de la conducta alimentaria: Influencia sobre el modelo estético corporal en púberes de ambos sexos. Psicol Social México. 2002; 9: 129-134.

16. Saucedo-Molina T. Modelos predictivos de dieta restringida en púberes, hombres y mujeres y en sus madres (Tesis de doctorado). Universidad Nacional Autónoma de México, México, 2003.

17. Palazzoli Selvini M. Cirillo S. Selvini M. y Sorrentino A.M. Muchachas anoréxicas y bulímicas. Paidós, Barcelona, 1999.

18. Matsanuga H. Kaye W.H. McConaha C. Personality disorders among subjects recovered from eating disorders. Int J Eat Disord 2000;27(3): 353-357.

19. Díaz Marsá M. Carrasco P.J. Sáiz Ruiz J. Decreased platelet MAO activity in female anorexia nervosa. Acta Psychiatr Scand 2000; 101:1-5.

20. Díaz Marsá M. Carrasco P.J. La personalidad y sus trastornos en la anorexia y en la bulimia nerviosa. En Sáiz Ruiz,J. y García-Camba M.E. Avances en trastornos de la conducta alimentaria. Anorexia nerviosa, bulimia nerviosa, obesidad. Barcelona: Masson 2002, p. 93-106.

21. Hsu, G.K.L. Can dieting cause an eating disorder? Psicol Med 1997; 27: 509-513.

22. González S.L.M., Lizano, M.M. y Gómez Pérez-
Mitré G. Factores de Riesgo en Desórdenes del Comer: Hábitos Alimentarios y Auto-atribución en una muestra de Niños Escolares Mexicanos. Rev Mex Psicol 1999; 16 (1): 117-126.

23. Waaddegaard M. Dieting and desire for weight loss among adolescents in Denmark: Aquestionnaire survey. Eur Eat Disord Rev 2002; 5(10): 329-346.

24. Gómez Pérez-Mitré G. Prevención primaria: Autodiagnóstico de factores de riesgo para el desarrollo de trastornos de la alimentación y de la nutrición. Informe Proyecto IN305599 (PAPIIT) y 34507-H (CONACyT) México, DF: Universidad Nacional Autónoma de México; 2001.

25. Garner, D, y Garfinkel, P. Handbook of psychotherapy for Anorexia Nervosa and Bulimia. New York: Guilford Press. 1985.

26. Gómez Pérez-Mitré, G. Alteraciones de la imagen corporal en una muestra de escolares mexicanas púberes. Rev Mex Psico 1997; 14: 31-40.

27. Gómez Pérez-Mitré G. Saucedo-Molina T. Ávila A.E. Factores de riesgo: anomalías de la conducta alimentaria en escolares preadolescentes. Congreso Regional de Psicología para profesionales de América. 1997.

28. Keefe K. Perceptions of normative social pressure and attitudes toward alcohol use: Changes during adolescence. J Studies Alcohol 1994; 55: 46-54.

29. Neumark-Sztainer D. Story M. Resnick M.D. Blum W.R. Lessons learned about adolescent nutrition from the Minnesota Adolescent Health Survey. J Am Dietetic Assoc 1998; 96 (12):1449-56.

30. Gustafson-Larson A.M. Rhonda D.T. Weight-related behaviors and concerns of fourth-grade children. $\mathrm{J}$ Am Dietetic Assoc 1992; 92: 818-22.

31. Hill J.A. Pre-adolescent dieting: implications for eating disorders. J Rev Psychiatry 1993; 5: 87-100.

32. González S.L.M. Lizano M.M. Gómez Pérez-Mitré G. Factores de Riesgo en Desórdenes del Comer: Hábitos Alimentarios y Auto-atribución en una muestra de Niños Escolares Mexicanos. Rev Mex Psicol 1999; 16 (1): 117-126.

33. Gómez Pérez-Mitré G. Ávila A.E. ¿Los escolares mexicanos hacen dieta con propósitos de control de peso? Rev Iberoam 1998; 6: 37-46.

34. Van Serien T. Frijters J.E.R. Berger G.P.A. Defares, P.B. The Dutch Eating Behavior Questionnaire (DEBQ) for assessment of restrained, emotional, and external eating behaviour. Int $\mathrm{J}$ Eat Disord 1986; 5: 295-315.

35. Edlund B. Halvarson K. Sjödén P-O. Eating behaviours and attitudes to eating, dieting, and body image in 7-year-old Swedish girls. Eur Eat Disorders Rev 
1996; 4: 40-53.

36. Hill A.J. Oliver S. y Rogers P.J. Eating in the adult world: The rise of dieting in childhood. British J Clin Pshychol 1992; 30: 265-267.

37. Halvarsson K. Sjödén P-O. Psychometric Properties of the Dutch Eating Behaviour Questionnaire (DEBQ) Among 9-10-Year-Old Swedish Girls. Eur Eat Disord Rev 1998; 6: 115-125.

38. Archer L. Rosembaum PL Streiner D. The Children's Eating Behavior Inventory: Reliability and Validity Results. J Pediatric Pshychol 1991; 16(5): 629-642.

39. Maloney M.J. McGuire J.B. Daniels S.R. Reliability testing of a children's version of the Eating Attitudes Test. J Am Acadm Child Adols Psychiatry 1988; 27: 541-543.

40. Garner D.M. Garfinkel P.E. The Eating Attitudes Test: An index of the symptoms of anorexia nervosa. Psychol Med 1979; 19: 273-279.

41. Kelly C. Ricciardelli K.L. y Clarke J.D. Disordered eating in adolescent males from a school based sample. Int J Eat Disord 1999; 25: 281-286.

42. Childress A.C. Brewerton T.D. Hodges E.L. Jarrel M.P. The Kid's Eating Disorders Survey (KEDS): A study of middle school students. J Am Acadm Child Adols Psychiatry. 1993; 32: 843-850.

43. Gómez Pérez-Mitré G. Cuestionario de Alimentación y Salud. Estudios de validez: alteraciones de la alimentación. CONACyT 300898. México. 1998.

44. Gómez Pérez-Mitré, G. Escala de factores de riesgo asociados a trastornos de la conducta alimentaria. Prevención primaria y factores de riesgo en trastornos alimentarios. IN-305599(PAPIT) UNAM. 34507-H CONACyT. México. 2000.

45. Toro J. Salamero M. Martínez E. Assessment of sociocultural influences on the aesthetic body shape model in anorexia nervosa. Acta Psychiat Scand 1994; 89: 147-151.
46. Tanner J.M. Growth at Adolescence. Blackwell. USA. 1962.

47. Reglamento de la Ley General de Salud en Materia de Investigación para la Salud. Rev Salud Pública Nutr 2003; 4(3).

48. SPSS, Inc.: Paquete Estadístico para las Ciencias Sociales (SPSS) para Windows versión 11 en español (Software de computadora) Chicago, Ill, SPSS, Inc, 2000.

49. Hair F.J. Anderson E.R. Tatham L.R. Black C.W. Análisis multivariante. $5^{\mathrm{a}}$ ed. Prentice Hall Iberia, Madrid, 1999.

50. Huon G.F. Health promotion and the prevention of dieting-induced disorders. In J Eat Disord 1996; 4(1):27-32.

51. Toro J. El cuerpo como delito. Anorexia, bulimia, cultura y sociedad. Barcelona, España: Editorial Ariel. 1996.

52. Saucedo-Molina T Gómez Peresmitré G. Modelo predictivo de dieta restringida en púberes mexicanas. Rev Psiquiatría Fac Med Barna 2004; 30(2):6974.

53. Saucedo-Molina T. Gómez Pérez-Mitré G. Modelo predictivo de dieta restringida en varones mexicanos. Rev Psiquiatría Fac Med Barna 2005; 32(2):6774.

54. Gardner R.M. Assessment of Body Image Disturbance in Children and Adolescents. En J. Kevin Thompson y L. Smolak (Eds.) Body Image, Eating Disorders, and Obesity in Youth. Assessmet, Prevention, and Treatment. Washington D.C, American Pshychological Association. 2001, pp.193-213

55. Saucedo-Molina T. Guzmán Saldaña R. Validez y confiabilidad de un instrumento para evaluar factores de riesgo asociados a trastornos alimentarios en púberes. Resultados preliminares. En Evaluación en psicología: del individuo a la interacción. Universidad Autónoma del Estado de Hidalgo (en prensa). 